\title{
The role of exercise combined with tocilizumab in visceral and epicardial adipose tissue and gastric emptying rate in abdominally obese participants: protocol for a randomised controlled trial
}

Regitse Højgaard Christensen ${ }^{*}$, Anne-Sophie Wedell-Neergaard ${ }^{1}$, Louise Lang Lehrskov' ${ }^{1}$ Grit Elster Legård ${ }^{1}$, Emma Berndt Dorph', Stine Nymand ${ }^{1}$, Maria Korf Ball ${ }^{1}$, Morten Zacho ${ }^{1}$, Robin Christensen ${ }^{3,5}$, Helga Ellingsgaard ${ }^{1}$, Jaya Birgitte Rosenmeier ${ }^{2}$, Rikke Krogh-Madsen', Bente Klarlund Pedersen ${ }^{1}$ and Kristian Karstoft ${ }^{1,4}$

\begin{abstract}
Background: Exercise reduces the amount of visceral adipose tissue (VAT) and the risk of cardiometabolic diseases. The underlying mechanisms responsible for these exercise-induced adaptations are unclear, but they may involve lipolytic actions of interleukin-6 (IL-6). Contracting skeletal muscles secrete IL-6, leading to increased circulating IL-6 levels in response to exercise. The aim of this study is to investigate whether IL-6 is involved in mediating the effects of exercise on visceral and epicardial adipose tissue volume and glycaemic control.

Methods/design: Seventy-five physically inactive males and females aged $>18$ years with a waist-to-height ratio $>0.5$ and/or waist circumference $\geq 88 \mathrm{~cm}$ (females) or $\geq 102 \mathrm{~cm}$ (males) are being recruited to participate in a 12-week intervention study. Participants are randomly allocated to one of five groups (1:1:1:1:1). Two groups consist of supervised endurance exercise training combined with the IL-6 blocker tocilizumab (ET) or saline used as placebo (EP), two groups consist of no exercise combined with tocilizumab (NT) or placebo (NP), and one group consists of resistance exercise and placebo (RP). Although the study is an exploratory trial, the primary outcome is change in VAT volume from before to after intervention, with secondary outcomes being changes in (1) epicardial adipose tissue, (2) pericardial adipose tissue and (3) gastric emptying. Depots of adipose tissue are quantitated by magnetic resonance imaging Gastric emptying and glucose metabolism are assessed using mixed-meal tolerance tests.
\end{abstract}

Discussion: Understanding the role of IL-6 in mediating the effects of exercise on visceral and epicardial adipose tissue and glycaemic control may lead to novel therapeutic approaches in the prevention of cardiometabolic diseases.

Trial registration: ClinicalTrials.gov, NCT02901496. Registered on 1 August 2016 and posted retrospectively on 15 September 2016.

Keywords: Visceral adipose tissue, Epicardial adipose tissue, Glucose homeostasis, Gastric emptying rate, Interleukin-6, Exercise

\footnotetext{
* Correspondence: regitse.hoejgaard.christensen@regionh.dk

${ }^{1}$ The Centre of Inflammation and Metabolism (CIM) and The Centre for Physical Activity Research (CFAS), Rigshospitalet, University of Copenhagen, Blegdamsvej 9, DK-2100 Copenhagen, Denmark

Full list of author information is available at the end of the article
}

(C) The Author(s). 2018 Open Access This article is distributed under the terms of the Creative Commons Attribution 4.0 International License (http://creativecommons.org/licenses/by/4.0/), which permits unrestricted use, distribution, and reproduction in any medium, provided you give appropriate credit to the original author(s) and the source, provide a link to the Creative Commons license, and indicate if changes were made. The Creative Commons Public Domain Dedication waiver (http://creativecommons.org/publicdomain/zero/1.0/) applies to the data made available in this article, unless otherwise stated. 


\section{Background}

Cardiometabolic diseases are a leading cause of death worldwide and are becoming a dominant health problem globally. Common pathogenic features shared by cardiometabolic diseases are visceral and cardiac adiposity, low-grade inflammation and impaired glycaemic control $[1,2]$. It is well known that regular endurance exercise reduces the volume of visceral adipose tissue (VAT) and improves glycaemic control, and also overall lowers the risk of cardiometabolic disease substantially [3-6].

The underlying mechanisms driving the exercise effects on VAT lipid metabolism are poorly understood, but they may involve the cytokine interleukin-6 (IL-6). During intensive exercise, skeletal muscles secrete IL-6 into the circulation $[1,7]$. IL- 6 has been identified as a lipolytic agent, and a single infusion of recombinant human IL-6 at physiological concentrations increases lipolysis and fat oxidation in humans [8]. This finding was replicated in vitro, with myotubes and adipocytes increasing fat oxidation and lipolysis following IL-6 stimulation [8]. Whether the exercise-induced reductions in VAT are caused by the lipolytic effects of IL-6 or other exercise factors is not known.

Whereas substantial evidence exists regarding the training-induced reductions in VAT, the effect of exercise on cardiac adipose tissue is poorly elucidated. One study has shown that endurance exercise causes a reduction in epicardial adipose tissue thickness [9]. Yet, it is unknown whether the exercise-mediated reduction in visceral fat is equipotent in epi- and pericardial fat (EAT and PAT, respectively). In addition, whether resistance exercise can similarly reduce EAT and PAT volume is unknown. Furthermore, whether the mechanism driving the possible effects of exercise on EAT and PAT involves IL-6 signalling remains to be investigated.

Despite an extensive amount of research on IL-6 in rodents, it remains to be investigated how repetitive systemic spikes of IL-6 (as seen with exercise) affect glycaemic control in humans. On the basis of studies in mice, it is known that IL-6 improves glucose tolerance through a glucagon-like peptide-1 (GLP-1)-mediated increase in insulin secretion [10]. In humans, an acute increase in circulating IL-6 improves glycaemic control by delaying the rate of gastric emptying (in press [11]). In humans, gastric emptying rate is a critical regulator of glycaemic control [12]. Yet, it remains to be investigated whether repetitive systemic spikes of muscle-derived IL-6 influence glycaemic control through changes in gastric emptying rate.

\section{Methods/design}

\section{Study hypotheses and aims}

We hypothesise that endurance exercise plus saline (placebo) is superior in mediating the reduction in visceral, epi- and pericardial adiposity and is superior in delaying gastric emptying compared with endurance exercise plus tocilizumab (IL-6 blocking agent); that is, if the null hypothesis is rejected and there is a difference between the two endurance exercise groups, the alternative hypothesis might be true.

The primary aim of this study is to determine whether blocking IL-6 signalling will ameliorate the endurance exercise-mediated reductions in VAT volume. Secondary aims are to investigate the importance of endurance exercise-induced increases in IL-6 on EAT thickness/ area/volume, glucose homeostasis and gastric emptying. Because it is not established if circulating levels of IL-6 are increased in response to resistance training [13, 14], it was not within the scope of this study to include a resistance exercise group receiving tocilizumab. However, as a separate distinctive aim, the resistance exercise plus placebo group was included to investigate the effects of resistance exercise vs. endurance exercise on VAT, EAT and glucose homeostasis.

\section{Study design and study setting}

The study is designed as an exploratory, double-blind, randomised, placebo-controlled trial consisting of 12 weeks of endurance exercise $(\mathrm{E})$ or no exercise $(\mathrm{N})$ combined with monthly infusions of tocilizumab (T) or placebo (P), resulting in five groups (ET, EP, NT, NP and RP), where RP is a placebo group using resistance exercise rather than endurance exercise. A total of 75 physically inactive participants are randomised in a ratio of 1:1:1:1:1 to exercise groups or control groups in combination with tocilizumab or saline, as depicted in Fig. 1. The enrolment period began on 1 August 2016 and was estimated to be completed by 26 April 2018. All baseline and follow-up tests are carried out at the Centre for Physical Activity Research (CFAS), Rigshospitalet, Copenhagen, Denmark, and the MRI department of Frederiksberg Hospital, Frederiksberg, Denmark.

\section{Participants \\ Eligibility}

Participants are recruited through advertisements in local newspapers and via social media. Eligibility criteria are listed in Table 1. Initial inclusion and exclusion criteria are identified through email and phone interview. Participants are eligible if they are white, aged 1880 years, abdominally obese and physically inactive (Table 1). Participants of same race are chosen in order to limit the effect of racial background on lipid and glucose metabolism [15]. The broad age span is chosen because IL-6 response to exercise is not known to be age-dependent [16, 17]. Abdominal obesity, which is indicative of visceral adiposity, is defined as a waist circumference $>88 \mathrm{~cm}$ for women or $102 \mathrm{~cm}$ for men $[18,19]$. Physical inactivity is defined as no engagement 


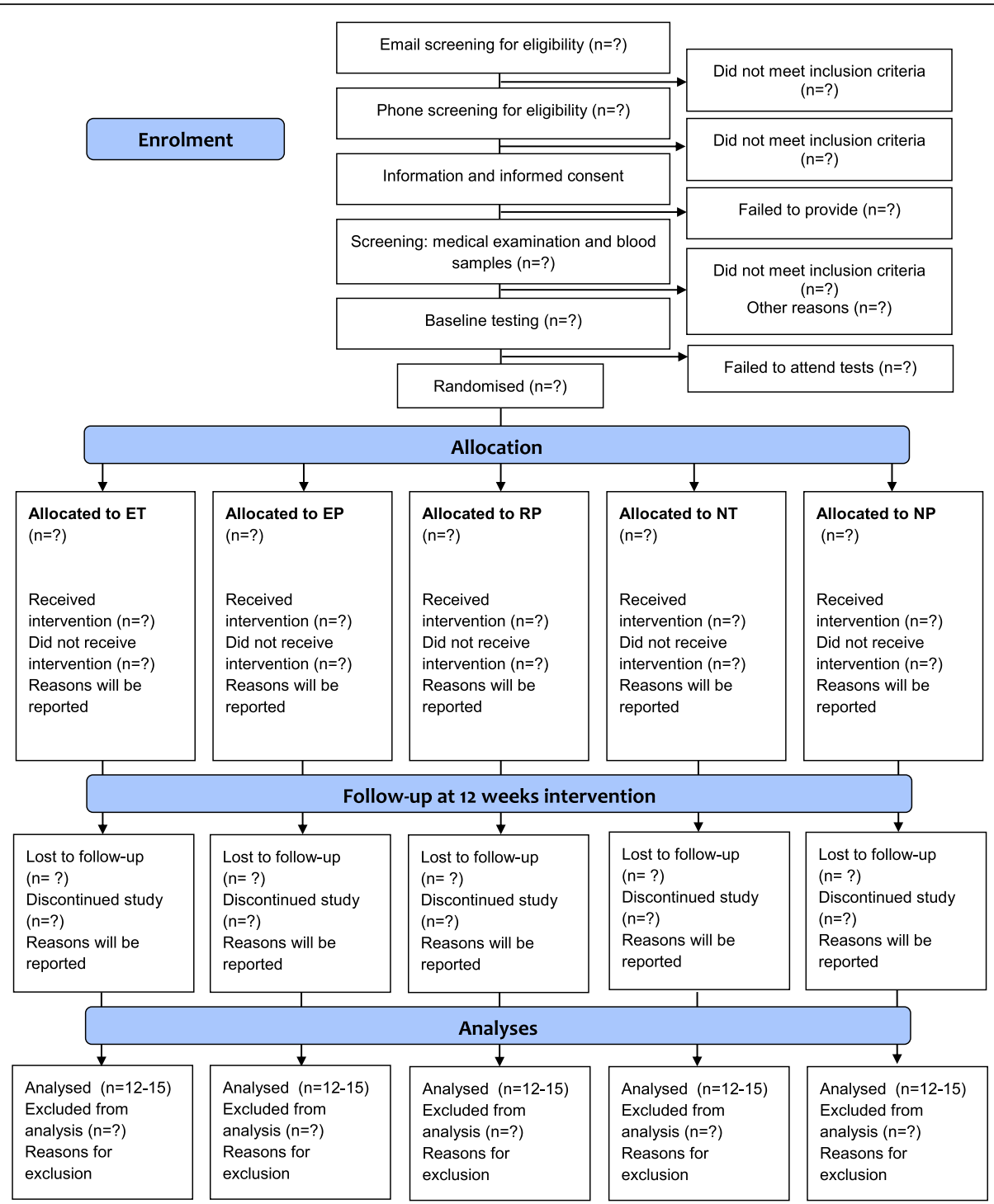

Fig. 1 Flow of participants through the study. Any reasons for discontinuation or exclusion from analysis are reported. ET (Endurance exercise + tocilizumab), EP (Endurance exercise + saline (placebo)), RP (Resistance exercise + saline), NT (No-exercise control + tocilizumab), NP (No-exercise control + saline)

Table 1 Eligibility of study participants

\begin{tabular}{|c|c|}
\hline Inclusion criteria & Exclusion criteria \\
\hline $\begin{array}{l}\text { 1. Age } 18-80 \text { yr } \\
\text { 2. White race } \\
\text { 3. Physically inactive (defined as not meeting the current guidelines of } \\
2.5 \text { h of physical activity per wk) } \\
\text { 4. Waist-to-height ratio } \geq 1: 2 \text { and/or waist circumference } \geq 88 / 102 \mathrm{~cm} \\
\text { (for women/men) } \\
\text { 5. Permitted medicine should be taken at a stable dose during at least } \\
4 \text { wk prior to randomization and preferably remaining stable during } \\
\text { the study. }\end{array}$ & $\begin{array}{l}\text { 1. Diabetes }(\mathrm{HbA} 1 \mathrm{c} \geq 48 \mathrm{mmol} / \mathrm{mol} \text { or fasting glucose } \geq 7.0 \mathrm{mmol} / \mathrm{L}) \\
\text { 2. Pregnancy } \\
\text { 3. Ischaemic heart disease } \\
\text { 4. Atrial fibrillation } \\
\text { 5. Treatment with: } \\
\text { a. NSAIDs on a daily basis } \\
\text { b. Biologic rheumatic drugs } \\
\text { c. Systemic prednisolone } \\
\text { d. Other immunotherapy } \\
\text { 6. Health conditions that prevent individuals from participating in the } \\
\text { exercise training intervention } \\
\text { 7. Subjects who cannot undergo MRI scans (e.g., owing to kidney disease, } \\
\text { metallic implants or claustrophobia) }\end{array}$ \\
\hline
\end{tabular}


in any intentional leisure exercise activities and failure to meet the current American College of Sports Medicine (ACSM) exercise guidelines of $2.5 \mathrm{~h} / \mathrm{wk}[20,21]$ by unintentional physical activity associated with daily activities. The unintentional daily activity level is evaluated individually at the telephone screen by asking a number of questions, including (1) type of occupation, which should be sedentary (defined by primarily computerbased activities [20, 22]); (2) way of commuting, which should preferably be passive or alternatively biking at a slow pace $<30 \mathrm{~min} / \mathrm{d}$ on a maximum of $5 \mathrm{~d} / \mathrm{wk}$; and (3) the total daily activity level assessed by steps per day should be $<10,000$, which is a reliable threshold for meeting current activity guidelines [23]. If eligible after the phone interview, the participant provides informed oral and written consent and subsequently undergoes a medical screening that includes blood sampling in order to identify latent exclusion criteria.

\section{Interventions}

The intervention is a combination of two components: a medical intervention (tocilizumab $[\mathrm{T}]$ or placebo $[\mathrm{P}]$ ) and a lifestyle intervention (supervised exercise [E] or no $[\mathrm{N}]$ exercise) as detailed below. The resulting intervention and control groups are depicted in Fig. 1.

\section{Medical intervention: IL-6 antagonism (tocilizumab)}

Tocilizumab (RoActemra ${ }^{\circ}$; Roche, Basel, Switzerland) is a humanised monoclonal antibody against the IL- 6 receptor currently used for treatment of patients with moderate to severe rheumatoid arthritis [24]. Participants will receive an intravenous infusion of $8 \mathrm{mg} / \mathrm{kg}$ (maximal $800 \mathrm{mg}$ ) administered every 4 weeks during the 12-week intervention (a total of three times) [25]. We chose to give the maximal recommended dose of tocilizumab [24] because we aimed for maximal suppression of the effects of exercise-induced IL-6 secretion. Tocilizumab is generally well tolerated. Serious adverse reactions are rare but include infections and hypersensitivity reactions. Tocilizumab is purchased via the pharmacy at Rigshospitalet. It is prepared according to the recommendation $(8 \mathrm{mg} / \mathrm{kg}$ ) and diluted in $\mathrm{NaCl} 0.9 \%$ to a total volume of $100 \mathrm{ml}$. Qualified staff at CFAS are responsible for this procedure. The placebo consists of $100 \mathrm{ml}$ of $\mathrm{NaCl} 0.9 \%$. Both placebo and tocilizumab are administered via intravenous infusion at a flow rate of $2 \mathrm{ml} / \mathrm{min}$, and the infusion is concealed to maintain double blinding.

\section{Exercise intervention and lifestyle monitoring}

The exercise programme includes three weekly training sessions of $45 \mathrm{~min} /$ session over a 12-week period. Each session will be moderate to vigorous in intensity. The exercise programme follows current ACSM guidelines for vigorous exercise [21] and was chosen because exercise of similar intensity and duration is known to result in IL-6 release [1, 17] and lead to changes in VAT (the primary outcome) [13]. Training takes place at the Centre for Physical Activity Research CFAS using TechnoGym equipment (Pedan A/S, Copenhagen, Denmark) and software programs which allow monitoring and registration of all exercise sessions. All exercise sessions are supervised. Maximal oxygen consumption rate $\left(\mathrm{VO}_{2} \max \right)$ and onerepetition maximum (RM) of two resistance exercises (knee extension and chest press) are determined at baseline and after the intervention for each participant. To control for factors that may influence the effect of the interventions, participants are instructed not to change their lifestyle during the intervention, with the exception of the intervention. A self-reported 3-day dietary intake record is obtained monthly during the intervention period. Furthermore, free-living physical activity is measured monthly using axial accelerometer-based physical activity monitors (AX3; Axivity, Newcastle upon Tyne, UK) for a 4-day period. The monthly registrations are undertaken in relation to pre- and mid-testing as well as at post-testing on identical visits and on identical consecutive weekdays throughout the intervention to ensure comparable measurements.

\section{Endurance exercise}

High-intensity interval endurance exercise is performed on an ergometer bike. The intensity of the intervals progressively increases during the 12 weeks of training. The 45-minute exercise sessions consist of 8 minutes of warmup at $40 \%$ and $60 \%$ of $\mathrm{VO}_{2}$ max followed by 37 minutes of interval exercise at $75-85 \%$ of $\mathrm{VO}_{2}$ max, as shown in Fig. 2 .

\section{Resistance exercise}

The resistance exercise is matched to the endurance training with a duration of 45 minutes per session. The exercise is interval-type, medium-load, high-repetition, time-based resistance exercise. Participants perform ten exercises paired in five blocks. Three to five sets with ten to fifteen repetitions lasting 1 minute are performed. The aim is to reach complete exhaustion after each exercise. The specific resistance exercise load comprises $60-80 \%$ of 1 RM with progression during the 12 weeks. Ten different exercises are performed: shoulder press, leg extension, pull exercise, crunches, leg press, shoulder laterals, chest press, biceps, dumbbell squats and back extensions. Details of the resistance exercise protocol are depicted in Fig. 3.

\section{Outcomes}

Outcome variables are listed in Table 2.

\section{Primary outcome}

The primary outcome is the change in volume of VAT (measured using MRI) from baseline to after the 12-week 


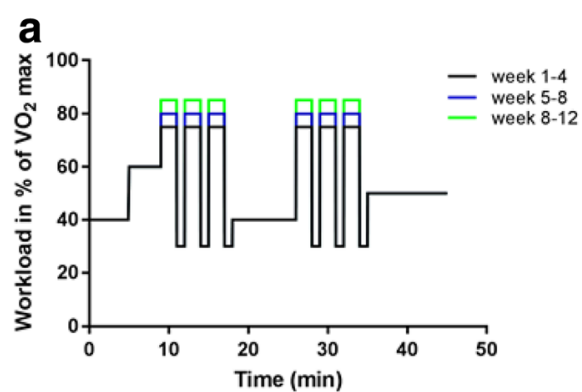

b

\begin{tabular}{|c|c|}
\hline \multicolumn{2}{|c|}{ Endurance exercise training programme details } \\
\hline Time (min) & Week 1-4 \\
\hline $0-4 \min$ & $40 \%$ of $V O 2 \max$ \\
\hline $5-8 \mathrm{~min}$ & $60 \%$ of $\mathrm{VO} 2 \max$ \\
\hline $9-17 \mathrm{~min}$ & $\begin{array}{l}3 \times 2 \text { min at } 75-85 \% \text { of } V O 2 \max , \\
1 \text { min recovery at } 30 \% \text { of } V O 2 \max \end{array}$ \\
\hline $18-25 \mathrm{~min}$ & $40 \%$ of $\mathrm{VO} 2 \max$ \\
\hline $26-34 \mathrm{~min}$ & $\begin{array}{l}3 \times 2 \text { min at } 75-85 \% \text { of } V O 2 \max \\
1 \text { min recovery at } 30 \% \text { of } V O 2 \max \end{array}$ \\
\hline $35-45 \mathrm{~min}$ & $50 \%$ of $\mathrm{VO} 2 \max$ \\
\hline
\end{tabular}

Fig. 2 Protocol of the endurance exercise. a Graphic demonstration of the different workload intervals of the endurance exercise programme. The workload is defined by the corresponding maximal oxygen consumption rate $\left(\mathrm{VO}_{2}\right.$ max) percentage. $\mathbf{b}$ Details of the exercise programme

intervention period, with comparison of the groups (Fig. 1).

\section{Secondary outcomes}

The secondary outcomes are changes in (1) EAT, (2) PAT and (3) gastric emptying. Also, we will explore the effects related to the interventions in regard to changes and alterations in rate of glucose homeostasis and lowgrade inflammation.

\section{Measurement of outcome variables}

The time points at which the outcomes are assessed at baseline, during the intervention and at follow-up are depicted in Fig. 4. The study is initiated with a clinical examination followed by baseline testing (Fig. 4, visits 1-4). Monthly tocilizumab/saline (placebo) infusions
(Fig. 4, visits 4-6) are administered during the intervention period. After the intervention period (12 weeks), the participants undergo follow-up testing (Fig. 4, visits $7-10)$ identical to the baseline tests.

\section{Blood samples}

Following an overnight fast (10 hours), blood samples, as specified in Fig. 4, are collected and processed by a trained laboratory technician and analysed according to standard procedures. Plasma is stored at $-80{ }^{\circ} \mathrm{C}$ prior to analysis.

\section{Abdominal and cardiac magnetic resonance imaging}

MRI is performed at Frederiksberg Hospital using a 1.5-Tesla whole-body MRI scanner (Ingenia; Philips, Eindhoven, the Netherlands). Abdominal MRI scans are

a

\begin{tabular}{|l|l|l|l|l|l|l|l|l|l|l|l|l|}
\hline \multicolumn{10}{|l|}{ Resistance exercise training protocol to be performed 3 times weekly } \\
\hline Weeks & 1 & 2 & 3 & 4 & 5 & 6 & 7 & 8 & 9 & 10 & 11 & 12 \\
\hline Set of each block & 3 & 3 & 3 & 3 & 3 & 3 & 3 & 3 & 4 & 4 & 4 & 4 \\
\hline Repetitions in set & 10 & 12 & 15 & 15 & 12 & 12 & 12 & 12 & 10 & 10 & 10 & 10 \\
\hline \% RM & 60 & 60 & 60 & 60 & 70 & 70 & 70 & 70 & 75 & 75 & 75 & 75 \\
\hline
\end{tabular}

b

\begin{tabular}{|c|c|c|c|c|}
\hline \multicolumn{2}{|l|}{ Exercises and blocks in the resistance programme } \\
\hline Block 1 & Block 2 & Block 3 & Block 4 & Block 5 \\
\hline Shoulder Press & Leg Extension & Leg Press & Chest Press & Dumbell Squats \\
\hline Horisontal Pull & Crunches & Shoulder Laterals & Biceps & Back Extensions \\
\hline
\end{tabular}

Fig. 3 Overview of the resistance exercise protocol. a Progression in frequency of sets and repetitions during the 12-week exercise intervention performed $3 \times$ weekly. $\mathbf{b}$ The ten individual exercises that are paired in five blocks and performed in each resistance exercise session with a duration of $45 \mathrm{~min} / \mathrm{session}$ 
Table 2 Outcome domains and measures assessed at baseline and follow-up (12 weeks after baseline)

\begin{tabular}{|c|c|c|}
\hline Outcome & Domain & Measurement \\
\hline Primary & Visceral fat tissue volume & MRI \\
\hline \multirow[t]{2}{*}{ Secondary } & $\begin{array}{l}\text { Epicardial adipose tissue } \\
\text { thickness/area/volume }\end{array}$ & Cardiac MRI \\
\hline & $\begin{array}{l}\text { Pericardial adipose tissue } \\
\text { thickness/ area/volume } \\
\text { Gastric emptying rate }\end{array}$ & $\begin{array}{l}\text { Cardiac MRI } \\
\text { MMTT and PCM }\end{array}$ \\
\hline \multirow[t]{12}{*}{ Other } & Total body fat mass & DXA \\
\hline & Total body muscle mass & DXA \\
\hline & Resting blood pressure & BP monitor \\
\hline & Heart rate recovery & Heart rate strap \\
\hline & $\begin{array}{l}\text { Maximal aerobic capacity } \\
\text { (cardiovascular fitness) }\end{array}$ & $\mathrm{VO}_{2}$ max test \\
\hline & Muscle strength & One-RM test \\
\hline & $\begin{array}{l}\text { Diabetes, pre-diabetes } \\
\text { and glucose tolerance }\end{array}$ & OGTT \\
\hline & $\begin{array}{l}\text { Glycaemic control during } \\
\text { mixed-meal tolerance test }\end{array}$ & MMTT \\
\hline & $\begin{array}{l}\text { Free-living glycaemic } \\
\text { control }\end{array}$ & CGM \\
\hline & Acute exercise bout $^{\mathrm{a}}$ & Blood tests \\
\hline & Low-grade inflammation ${ }^{\mathrm{b}}$ & Blood tests \\
\hline & $\begin{array}{l}\text { Cardiometabolic } \\
\text { biomarkers }{ }^{\mathrm{C}} \text { and } \\
\text { adipokines }\end{array}$ & Blood tests \\
\hline
\end{tabular}

Abbreviations: CGM Continuous glucose monitoring, DXA Dual-energy X-ray absorptiometry, MMTT Mixed-meal tolerance test, MRI Magnetic resonance imaging, OGTT Oral glucose tolerance test, PCM Paracetamol, $R M$ Repetition maximum

${ }^{a}$ Blood will be analysed for exercise factors, including interleukin-6, immune cell counts and function, and biomarkers of low-grade inflammation ${ }^{\mathrm{b}}$ Blood will be analysed for biomarkers of low-grade inflammation, including high-sensitivity C-reactive protein, tumour necrosis factor-a, interferon- $\gamma$ and interleukins

'Blood will be analysed for cardiometabolic biomarkers, including total cholesterol, high-density lipoprotein, low-density lipoprotein, glycated haemoglobin, fasting insulin, fasting glucose and pro-brain natriuretic peptide

obtained as transverse images (slice thickness of $5 \mathrm{~mm}$ ) from the top of the thoracic diaphragm to the pelvic floor. VAT will be manually marked on each image, capturing the area from the diaphragm to the first sacral vertebra, and the volume will be calculated as the sum of all voxels using MANGO (Multi-Image Analysis GUI) version 2.5 [26, 27].

Cardiac MRI is supervised by a senior cardiologist, and sagittal, axial and horizontal planes are obtained from five retrospective cardiac cycles. Cardiac function including myocardial mass is quantified using cine imaging with a slice thickness of $8 \mathrm{~mm}$ (inter-slice gap, $2 \mathrm{~mm}$ for the short-axis stack) and a temporal resolution of 30-50 milliseconds, depending on heart rate (25 acquired phases). The number of short-axis cine images acquired is participant-specific, depending on ventricular size, using retrospective gating when possible in all participants.
Semi-automatic calculations using cardiovascular imaging software ${\left(\mathrm{cvi}^{42}\right.}^{4}$ version 5.2; Circle Cardiovascular Imaging, Calgary, AB, Canada) will be used for post-processing using endo- and epicardial contours to calculate left ventricular ejection fraction and cardiac muscle mass. EAT and PAT thicknesses, areas and volumes will be quantified from images in the end-diastolic four-chamber view and short-axis view. Contours around the fat layers of the ventricles and atria will be drawn manually. All MRI scans will be analysed in a blinded manner by trained medical doctors. A subset of the cardiac MRIs will be evaluated by a cardiologist to assess accuracy and inter-reader variability.

\section{Maximal aerobic capacity}

The participants undergo a graded exercise test on an ergometer bicycle for determination of $\mathrm{VO}_{2} \max$. The test starts with a 5-minute warm-up at $70 \mathrm{~W}$. Warm-up is immediately followed by a $15-\mathrm{W}$ increase every minute until exhaustion. Ventilation rate and expired $\mathrm{O}_{2}$ and $\mathrm{CO}_{2}$ levels are measured via an indirect calorimetric system, and heart rate is assessed simultaneously for the duration of the test. Two separate $\mathrm{VO}_{2}$ max tests are performed during the baseline tests (Fig. 4) to allow participants to familiarise themselves with the bicycle and the indirect calorimetric ventilation system. The test with the best performance is used to assess the changes in aerobic capacity from baseline to follow-up.

\section{Body composition}

Dual-energy $\mathrm{x}$-ray absorptiometry is used to assess total body fat mass and lean body mass one time before, two times during and one time after the intervention.

\section{Mixed-meal tolerance test}

The mixed meal consists of a 350-ml liquid drink containing $385 \mathrm{kcal}$. The energy distribution is $15 \%$ fat, $20 \%$ protein and $65 \%$ carbohydrate (of which $9 \%$ is glucose). To allow assessment of the rate of gastric emptying, 1.5 -g paracetamol is dissolved and added to the meal as a pharmacological tracer [28]. Participants are encouraged to ingest the meal in less than 2 minutes, and serial blood samples will be drawn -10, 0, 15, $30,60,90,120,150$ and 180 minutes after intake of the liquid meal. In addition to paracetamol, blood samples will be analysed for glucagon, GLP-1, GIP, PYY, insulin, pro-insulin, C-peptide and glucose.

\section{Oral glucose tolerance test}

A standard 2-hour 75-g oral glucose tolerance test is performed following an overnight fast (10 hours). Blood samples are drawn at baseline (0), 15, 30, 60 and 90 minutes and after 120 minutes, and then they are analysed for glucose, insulin, and C-peptide. 


\begin{tabular}{|c|c|c|c|c|c|c|c|c|c|c|}
\hline \multirow{2}{*}{$\begin{array}{l}\text { Timeline } \\
\text { Visit }\end{array}$} & \multicolumn{4}{|c|}{ Baseline } & \multicolumn{2}{|c|}{$\begin{array}{l}\text { Intervention } \\
\text { (12 weeks) }\end{array}$} & \multicolumn{4}{|c|}{ Follow-up } \\
\hline & 1 & 2 & 3 & 4 & 5 & 6 & 7 & 8 & 9 & 10 \\
\hline Study day (time points) & 1 & 2 & 3 & 4 & 30 & 60 & 90 & 91 & 92 & 93 \\
\hline \multicolumn{11}{|l|}{ Enrolment } \\
\hline Eligibility screen & $\mathrm{X}^{1}$ & & & & & & & & & \\
\hline Informed consent & $\mathbf{X}$ & & & & & & & & & \\
\hline Allocation & & & $\mathbf{X}$ & & & & & & & \\
\hline \multicolumn{11}{|l|}{ Interventions } \\
\hline Infusions of placebo/tocilizumab & & & & $\mathbf{X}$ & $\mathbf{X}$ & $\mathbf{X}$ & & & & \\
\hline Exercise $^{2} /$ no exercise control & & & & $\mathbf{X}$ & $\mathbf{X}$ & $\mathbf{X}$ & & & & \\
\hline \multicolumn{11}{|l|}{ Assessments } \\
\hline Medical history & $\mathrm{X}$ & & & & $\mathrm{X}$ & $\mathrm{X}$ & $\mathrm{X}$ & & & \\
\hline $\begin{array}{l}\text { Clinical examination } \\
\text { (Vital signs, BP, ECG) }\end{array}$ & $\mathrm{X}$ & & & & $\mathrm{X}$ & $\mathrm{X}$ & $\mathrm{X}$ & & & \\
\hline Blood test $^{3}$ & $\mathrm{X}$ & & $\mathrm{X}$ & $\mathrm{X}$ & $\mathrm{X}$ & $\mathrm{X}$ & $\mathrm{X}$ & & $\mathrm{X}$ & $\mathrm{X}$ \\
\hline $\begin{array}{l}\text { DXA scan and anthropometrics } \\
\text { (WC, HC, weight, BMI) }\end{array}$ & $\mathrm{X}$ & & & & $\mathrm{X}$ & $\mathrm{X}$ & $\mathrm{X}$ & & & \\
\hline $\mathrm{VO}_{2}$ max test & $\mathrm{X}$ & & $\mathrm{X}$ & & & & $\mathrm{X}$ & & & \\
\hline One RM test & $\mathrm{X}$ & & & & & & $\mathrm{X}$ & & & \\
\hline Abdominal and cardiac MRI & & $\mathrm{X}$ & & & & & & $\mathrm{X}$ & & \\
\hline Fat $+/$ - muscle biopsies & & & $\mathrm{X}$ & & & & & & $\mathrm{X}$ & \\
\hline OGTT & & & $\mathrm{X}$ & & & & & & $\mathrm{X}$ & \\
\hline Acute exercise bout & & & & $\mathrm{X}$ & & & & & & $\mathrm{X}$ \\
\hline Diet registration & & & & $\mathrm{X}$ & $\mathrm{X}$ & & & & & $\mathrm{X}$ \\
\hline Activity registration (AX3) & & & & $\mathrm{X}$ & $\mathrm{X}$ & & & & & $\mathrm{X}$ \\
\hline Continuous glucose monitoring & & & & $\mathrm{X}$ & & & & & & $\mathrm{X}$ \\
\hline
\end{tabular}

Fig. 4 Time schedule of enrolment, interventions and assessments. Abbreviations: BMI Body mass index, BP Blood pressure, DXA Dual-energy X-ray absorptiometry, ECG Electrocardiogram, MRI Magnetic resonance imaging, $H C$ Hip circumference, $V_{2}$ max Maximal oxygen consumption rate, WC Waist circumference, MMTT Mixed-meal tolerance test, OGTT Oral glucose tolerance test, RM Repetition maximum. 'Eligibility screen was conducted in a telephone interview before study day $1 .{ }^{2}$ The exercise intervention is a 12-week supervised exercise programme with three sessions per week consisting of either endurance or resistance exercise training. ${ }^{3}$ Blood samples will be drawn after an overnight fast (> 10 hours) and analysed for haematologic, renal, endocrine, cardiac and hepatologic markers. Table is adapted from the SPIRIT recommendations [38]

\section{Continuous glucose monitoring}

Three days of continuous glucose monitoring (CGM) is performed using enzyme-coated electrodes (iPro MMT7745WW; Medtronic, Northridge, CA, USA) placed subcutaneously in the abdominal wall. For calibration of the CGM system, finger-prick blood glucose measurements are performed by the participant four times daily.

\section{Acute bout of exercise}

For the acute bout of exercise, participants perform a 45-minute exercise bout similar to an exercise session. The workload is determined according to the latest individual watt- $\mathrm{VO}_{2}$ relationship assessed during the preand post $-\mathrm{VO}_{2}$ max test. Blood samples are drawn prior to, during and after the exercise bout (baseline and at 22, 45, 105 and 165 minutes) and analysed for IL-6, immune cell count and function, and other plasma biomarkers.

\section{Statistical power and sample size considerations}

For a two-sample pooled $t$ test of a normal mean difference with a two-sided significance level of 0.05, assuming a common SD of 265-g VAT, a sample size of 14 in each group has a power of $82.2 \%$ to detect a difference between the means -300 and $0 \mathrm{~g}$ VAT in the endurance exercise group + saline group (EP) and the endurance exercise + tocilizumab group (ET), respectively. The expected change in VAT is based on unpublished data from the CFAS. Fourteen participants will also be included in each of the control groups (NT and NP) and the resistance exercise + saline group (RP). Anticipating only a minor risk of attrition (over 12 weeks), a final sample size of 75 participants will be included in the study (anticipating 15 in each group).

\section{Analysis populations}

This study (2TRAIN) primarily follows a per-protocol design where participants are included in the analysis 
only if they have completed the trial and adhered to tocilizumab/placebo combined with exercise/no-exercise intervention according to the pre-specified protocol. This design was chosen because we are interested in measuring the physiological effect which is not influenced by lack of adherence, unlike the intention-to-treat (ITT) principle (with use of "conservative imputation") [29]. Using the per-protocol design enables us to assess the true experimental effect of the medical and exercise intervention on the primary endpoint [29]. Because nonadherers generally differ from adherers with regard to motivational and prognostic factors, this introduces a bias in the per-protocol design. Yet, this bias is limited because the aim of this study is exploratory, with the aim of assessing the mechanism driving the exercise effects, which requires adherence rather than evaluation of treatment efficacy where differential prognostic factors would confound the effect measures.

Patterns of non-adherence and other missing data will be investigated but will be assumed to be missing at random. In case of high non-adherence, high drop-out rate or skewed drop-out between intervention groups, a third-party biostatistician will perform analyses that adjust for such instances. Satisfactory adherence is defined as complete adherence to tocilizumab/placebo intervention and a minimum of $80 \%$ adherence to the exercise intervention/no-exercise control.

In addition, the ITT principle will be applied when necessary for the purpose of sensitivity analysis to assess the robustness of the primary analyses where imputation techniques (e.g., baseline observations carried forward) will be used to replace missing data [30]. Any discrepancies in data analysis between the methods will be resolved by a third-party biostatistician.

\section{Statistical analyses and reporting}

Like all clinical trials, exploratory studies should have clear and precise objectives. However, in contrast to confirmatory trials, the objectives of the exploratory 2TRAIN trial may not lead to simple tests of predefined hypotheses; that is, we will apply a more flexible approach to the statistical interpretation of the findings. As a consequence, a trial such as 2TRAIN cannot be the basis of a formal proof of efficacy, although it may contribute to the total body of relevant physiological evidence.

The results will be reported in accordance with the Consolidated Standards of Reporting Trials (CONSORT) $[31,32]$. Descriptive variables will be summarised by means of frequency distributions, means and SDs, or medians and IQRs, by group as well as by the total population. Categorical data will be summarised by numbers and percentages.

The primary null hypothesis is based on the comparison between participants randomised to endurance exercise with (ET) or without (EP) tocilizumab; $\mathrm{H}_{0}$ :
$\mu_{\mathrm{ET}}=\mu_{\mathrm{EP}}$. The overall study design is based on five groups (EP, ET, NT, NP and RP), and rather than looking only at the two according to the primary objective, we apply linear modelling based on generalised linear models (GLMs) in the analysis of the primary outcome. Analysis of covariance (ANCOVA) models will be performed, which produces several diagnostic measures, provides contrasts and estimates for customised hypothesis tests, and it provides tests for means adjusted for covariates (e.g. , least squares [LS] means adjusted for baseline variables). The GLM procedure handles models relating a continuous dependent variable to one or several independent variables. The independent variables can be either classification variables, which divide the observations into discrete groups (e.g., EP, ET, NT, NP and RP), or continuous variables (e.g., the level at baseline). We will analyse continuous outcomes using ANCOVA models, with a fixed factor for group (five levels) and adjust for the value at baseline. For each continuous outcome variable (after performing the overall ANCOVA model [five-group comparison]), we will obtain the $P$ value and group contrast for the difference between the LS means: ET vs. EP (in analogy to a two-sample $t$ test [33]), independent of what the overall ANCOVA model indicates. Also, results following the ANCOVA model will be expressed as estimates of the group difference in the various pairwise comparisons (including ET vs. EP) in the changes from baseline, with $95 \%$ CIs to represent the precision of the estimates. To assess the adequacy of the linear models describing the observed data, as well as to check the assumptions for the systematic and random parts of the models, we will investigate the model features via the predicted values and the residuals; that is, the residuals have to be normally distributed (around 0 ) and be independent of the predicted values.

All reported $P$ values will be two-sided and will not be adjusted for multiple comparisons (despite having ten potential tests for each outcome variable $[5 \times\{5-1\} / 2]$ ). Per default, we set the statistical significance at the conventional level of $0.05(P<0.05)$. All analyses will be performed using commercially available statistical software.

\section{Blinding}

The primary investigators are blinded to intervention. Outcome assessors are blinded to allocation at baseline and follow-up. Owing to the nature of the study, participants cannot be blinded to the exercise modality, but they are blinded to tocilizumab intervention. Interim analyses are not performed.

\section{Randomisation: sequence generation and allocation concealment}

Participants are randomly assigned to groups in a 1:1:1: 1:1 ratio using computer-generated block randomisation. 
Seven blocks of ten participants and a final block of five participants were planned. In order to achieve a balanced sample size of complete cases in each group, drop-outs occurring in the first six blocks were replaced in the subsequent block. If drop-outs are encountered in the seventh and eighth blocks, these are not replaced. The randomised sequence generation was generated by a researcher who is not involved in the testing and was delivered concealed to the technical personnel preparing the infusions. Infusions are delivered to the study investigators in a concealed manner to maintain blinding. The allocation information regarding exercise modality is given to the participants by a study assistant not involved in the randomisation or evaluation procedures. Exercise sessions are supervised by educated study assistants to maintain blinding of primary investigators with regard to exercise modality.

\section{Safety (based on collected adverse events)}

Before every infusion of tocilizumab/placebo, participants undergo a clinical examination and an interview performed by a medical doctor to assess health status, followed by collection of a blood sample to detect any adverse reactions (including drug-related side effects). The following parameters are measured: alanine aminotransferase, aspartate aminotransferase, creatinine, haemoglobin, thrombocytes, glucose, total cholesterol, low-density lipoprotein cholesterol, high-density lipoprotein cholesterol, triglyceride, high-sensitivity C-reactive protein, leucocytes and leucocyte types.

Intravenous infusion of gadolinium during cardiac MRI scan can rarely cause adverse allergic reactions, but the medical doctors present are educated to identify and handle such situations. Rarely, infections or temporary paraesthesias occur in the skin lesion that follows blood sampling or muscle/fat biopsies. Participants are informed about side effects and signs of infection. The participants are urged to contact the medical doctors involved in the trial at all times in case of an adverse reaction or other symptoms experienced during the study period. Any serious adverse event (life-threatening, fatal, expected, unexpected, or drug-related) will be reported to the scientific ethics committee at the Capital Region of Denmark and, if requested, to the Danish Medicines Agency.

\section{Discussion}

In this study, we are investigating the role of IL-6 in mediating exercise-induced adaptations in an abdominally overweight population. We anticipate the results to provide insight into the role of IL-6 in exercise-mediated changes in VAT, EAT and PAT and glucose homeostasis. Furthermore, we hope to gain valuable insight related to the exercise modalities that can be translated into efficient exercise programmes for subjects with increased risk for metabolic diseases. Understanding how exercise and IL-6 regulate VAT, EAT and PAT volumes and glucose homeostasis may inform novel therapeutic approaches in the prevention and treatment of cardiometabolic diseases.

The study is a randomised controlled trial, and both participants and primary investigators are blinded with respect to the medical intervention of tocilizumab and the outcome assessments. It is not possible to blind participants with respect to the exercise intervention, and this may introduce a bias. To limit this bias, participants are urged not to change their lifestyle during the intervention, except from what they are randomised to, because this would potentially influence the outcomes of the intervention. The eating habits and activity levels of the participants are regularly assessed by use of dietary records and axial accelerometer-based physical activity monitors. It is important to acknowledge that the resistance and endurance exercise protocols are matched only in duration. This is a limitation which should be kept in mind when interpreting the effects of the different exercise modalities. To compare the intensity of the different exercise modalities, we ask the participants to wear a heart rate monitor during both resistance and endurance exercise sessions. Exercise intensity is determined by percentage of $\mathrm{VO}_{2}$ max, and not by the peak power output, in line with previous studies by our group [34, 35]. Helgerud et al. [36] previously showed $\mathrm{VO}_{2}$ max to increase in response to high-intensity interval exercise training. Therefore, we believe that adjustment of exercise intensity according to percentage of $\mathrm{VO}_{2}$ max accurately ensures high exercise intensities throughout the intervention in this study. In addition, the supervised nature of the exercise protocol will ensure participant adherence and intensity and thereby improve the homogeneity of each intervention. The measurement of the primary outcome by MRI follows the gold standard and will enable accurate estimations of changes in the VAT, EAT and PAT depots.

The study includes five intervention groups (ET, EP, $\mathrm{NT}, \mathrm{NP}$ and RP), and the endurance exercise groups with and without tocilizumab (ET vs. EP) are of primary interest. In order to control for isolated effects of tocilizumab, two no-exercise control groups (NT and NP) were included as well. The inclusion of a resistance exercise group without tocilizumab (RP) was intended only for future ad hoc objectives, including comparisons of exercise modalities (endurance vs. resistance exercise without tocilizumab) on changes in VAT, EAT, PAT and gastric emptying.

This study is conducted in accordance with the guidelines of the regional ethics committee and the Declaration of Helsinki II [37]. All participants receive written and oral 
information about the study prior to inclusion and their provision of oral and written consent. The participants can discontinue participation in the study at all times with no obligation to provide a reason. Data and associated biological material are stored in an individual database and research biobank at Rigshospitalet. The biological material will be destroyed after a maximum of 10 years. Confidentiality of the participants is maintained by assigning participants a study number, keeping identifiers separate from the data, and storing data in a locked file and secure computer database. Scientific reports generated from the study will not contain information that would identify the participants.

The study protocol adheres to the Standard Protocol Items: Recommendations for Interventional Trials (SPIRIT) guidelines [38] (Additional file 1), and results of this study will be reported according to the CONSORT guidelines [31]. Negative, positive or inconclusive results will be disseminated in international peer-reviewed scientific journals at national and international conferences.

\section{Trial status}

The study was prospectively registered at ClinicalTrials.gov (NCT02901496) on 1 August 2016 and posted retrospectively on 15 September 2016. The recruitment period began on 1 August 2016 and was estimated to be completed by 26 April 2018.

\section{Additional file}

Additional file 1: SPIRIT 2013 checklist: recommended items to address in a clinical trial protocol and related documents. (DOC $131 \mathrm{~kb}$ )

\begin{abstract}
Abbreviations
ACSM: American College of Sports Medicine; ANCOVA: Analysis of covariance; BP: Blood pressure; CFAS: Centre for Physical Activity Research; CGM: Continuous glucose monitoring; CONSORT: Consolidated Standards of Reporting Trials; DXA: Dual-energy x-ray absorptiometry; EAT: Epicardial adipose tissue; ECG: Electrocardiogram; EP: Endurance exercise training and placebo; ET: Endurance exercise training combined with tocilizumab; GLM: Generalised linear model; GLP-1: Glucagon-like peptide-1; HbA1c: Glycated haemoglobin; HC: Hip circumference; IL-6: Interleukin-6; ITT: Intention to treat; LS: Least-squares means; MMTT: Mixed-meal tolerance test; MRI: Magnetic resonance imaging; NP: No exercise combined with placebo; NSAID: Non-steroidal anti-inflammatory drug; NT: No exercise combined with tocilizumab; OGTT: Oral glucose tolerance test; PAT: Pericardial adipose tissue; PCM: Paracetamol; RM: Repetition maximum; RP: Resistance exercise and placebo; SPIRIT: Standard Protocol Items: Recommendations for Interventional Trials; TNF-a: Tumour necrosis factor-a; VAT: Visceral adipose tissue; $\mathrm{VO}_{2}$ max: Maximal oxygen consumption rate; WC: Waist circumference
\end{abstract}

\section{Acknowledgements}

We acknowledge senior research associates Mathias Ried-Larsen and Jesper Frank Christensen at the Centre for Physical Activity for invaluable recommendations and feedback on the design and statistical plan of this study.

\section{Funding}

The work is supported by the Tryg Foundation, Denmark (grant number 101390). Salary to the primary investigators is supported by the Danish Heart Foundation (grant number 16-R107-A6704-22970) and the Danish Diabetes Academy.
Running costs are financially supported by an Aase and Ejnar Danielsen Foundation medical research grant (grant number 10-001271). The Musculoskeletal Statistics Unit, Parker Institute (RC), is supported by core grant OCAY-13-309 from the Oak Foundation.

\section{Authors' contributions}

RHC, ASWN, LLL, GEL, JBR, HE, MZ, RKM, BKP and KK made substantial contributions to the concept and design of the study. RC elaborated on the statistical design of the study. EBD, SN, MKB and MZ designed the exercise interventions. RHC, ASWN, LLL, EBD, HE, RKM, JBR, BKP and KK drafted the protocol. RHC was responsible for drafting the protocol manuscript and drafted the statistical analyses. RHC, ASWN, LLL, GEL, JBR, HE, EBD, SN, MKB, $M Z, R C, H E, R K M, B K P$ and KK revised the manuscript critically for important intellectual content. All authors read and gave their approval of the final manuscript to be published.

Ethics approval and consent to participate

The research ethics committee of the Capital Region of Denmark approved the study before study initiation ( $\mathrm{H}-16018062)$. Written and oral informed consent is obtained from all participants prior to enrolment in the study.

\section{Competing interests}

The authors declare that they have no competing interests.

\section{Publisher's Note}

Springer Nature remains neutral with regard to jurisdictional claims in published maps and institutional affiliations.

\section{Author details}

${ }^{1}$ The Centre of Inflammation and Metabolism (CIM) and The Centre for Physical Activity Research (CFAS), Rigshospitalet, University of Copenhagen, Blegdamsvej 9, DK-2100 Copenhagen, Denmark. ²Department of Cardiology, Copenhagen University Hospital Bispebjerg, Capital Region of Copenhagen, Copenhagen, Denmark. ${ }^{3}$ Musculoskeletal Statistics Unit, The Parker Institute, Bispebjerg and Frederiksberg Hospital, F, Copenhagen, Denmark. ${ }^{4}$ Department of Clinical Pharmacology, Bispebjerg Hospital, University of Copenhagen, Copenhagen, Denmark. ${ }^{5}$ Department of Rheumatology, Odense University Hospital, Odense, Denmark.

Received: 10 December 2017 Accepted: 10 April 2018

Published online: 02 May 2018

\section{References}

1. Pedersen BK. The anti-inflammatory effect of exercise: its role in diabetes and cardiovascular disease control. Essays Biochem. 2006;42:105-17.

2. Noyes AM, Dua K, Devadoss R, et al. Cardiac adipose tissue and its relationship to diabetes mellitus and cardiovascular disease. World J Diabetes. 2014;5:868-76.

3. Pedersen BK. The diseasome of physical inactivity - and the role of myokines in muscle-fat cross talk. J Physiol. 2009;587:5559-68.

4. Vissers D, Hens W, Taeymans J, et al. The effect of exercise on visceral adipose tissue in overweight adults: a systematic review and meta-analysis. PLoS One. 2013;8:e56415.

5. Ross R, Dagnone D, Jones PJH, et al. Diet-induced weight loss or exercise-induced weight loss in men. Ann Intern Med. 2000;133:92-103.

6. Holloszy JO. Exercise-induced increase in muscle insulin sensitivity. J Appl Physiol. 2005;99:338-43.

7. Peterson AM, Pedersen BK. The role of IL- 6 in mediating the anti-inflammatory effects of exercise. J Physiol Pharmacol. 2006;57:43-51.

8. Petersen EW, Carey AL, Sacchetti M, et al. Acute IL-6 treatment increases fatty acid turnover in elderly humans in vivo and in tissue culture in vitro. Am J Physiol Endocrinol Metab. 2005;288:E155-62.

9. Kim MK. Aerobic exercise training reduces epicardial fat in obese men. J Appl Physiol. 2008;106:5-11.

10. Ellingsgaard $H$, Hauselmann I, Schuler B, et al. Interleukin- 6 enhances insulin secretion by increasing glucagon-like peptide-1 secretion from $L$ cells and alpha cells. Nat Med. 2011;17:1481-9.

11. Lang Lehrskov L, Lyngbaek MP, Soederlund, et al. Interleukin-6 delays gastric emptying in humans with direct effects on glycemic control. Cell Metab. 2018. https://doi.org/10.1016/j.cmet.2018.04.008. 
12. Holst JJ, Gribble F, Horowitz M, et al. Roles of the gut in glucose homeostasis. Diabetes Care. 2016;39:884-92.

13. Donges CE, Duffield R, Drinkwater EJ. Effects of resistance or aerobic exercise training on interleukin-6, C-reactive protein, and body composition. Med Sci Sport Exerc. 2010;42:304-13.

14. Olson TP, Dengel DR, Leon AS, et al. Changes in inflammatory biomarkers following one-year of moderate resistance training in overweight women. Int J Obes. 2007;31:996-1003.

15. Osei K, Gaillard T. Disparities in cardiovascular disease and type 2 diabetes risk factors in blacks and whites: dissecting racial paradox of metabolic syndrome. Front Endocrinol (Lausanne). 2017;8:204.

16. Pedersen BK, Febbraio MA. Muscles, exercise and obesity: skeletal muscle as a secretory organ. Nat Rev Endocrinol. 2012;8:457-65.

17. Pedersen BK. Anti-inflammatory effects of exercise: role in diabetes and cardiovascular disease. Eur J Clin Investig. 2017;47:600-11.

18. Lean MEJ, Han TS, Morrison CE. Waist circumference as a measure for indicating need for weight management. BMJ. 1995;311:158-61.

19. Vega GL, Adams-Huet B, Peshock R, et al. Influence of body fat content and distribution on variation in metabolic risk. J Clin Endocrinol Metab. 2006;91:4459-66.

20. Tremblay MS, Aubert S, Barnes JD, et al. Sedentary Behavior Research Network (SBRN) - Terminology Consensus Project process and outcome. Int J Behav Nutr Phys Act. 2017;14:75.

21. Garber CE, Blissmer B, Deschenes MR, et al. Quantity and quality of exercise for developing and maintaining cardiorespiratory, musculoskeletal, and neuromotor fitness in apparently healthy adults: guidance for prescribing exercise. Med Sci Sports Exerc. 2011;43:1334-59.

22. Sedentary Behaviour Research Network (SBRN). Letter to the editor: Standardized use of the terms 'sedentary' and 'sedentary behaviours'. Ment Health Phys Act. 2013;6:55-6.

23. Wattanapisit A, Thanamee S. Evidence behind 10,000 steps walking. J Health Res. 2017:31:241-8.

24. European Medicines Agency (EMA). RoActemra: European Public Assessment Report. London: EMA; 2017. http://www.ema.europa.eu/ema/ index.jsp?curl=pages/medicines/human/medicines/000955/human_med_ 001042.jsp\&mid=WC0b01ac058001d124. Accessed 26 Apr 2018.

25. Smolen JS, Beaulieu A, Rubbert-Roth A, et al. Effect of interleukin-6 receptor inhibition with tocilizumab in patients with rheumatoid arthritis (OPTION study): a double-blind, placebo-controlled, randomised trial. Lancet. 2008:371:987-97.

26. Lancaster J, Ghiatas A, Alyassin A, et al. Measurement of abdominal fat with T1-weighted MR images. J Magn Reson Imaging. 1991;1:363-9.

27. Positano V, Gastaldelli A, Sironi AM, et al. An accurate and robust method for unsupervised assessment of abdominal fat by MRI. J Magn Reson Imaging. 2004;20:684-9.

28. Heading R, Nimmo J, Prescott $L$, et al. The dependence of paracetamol absorption on the rate of gastric emptying. Br J Pharmac. 1973;47:415-21.

29. Hernán MA, Robins JM. Per-protocol analyses of pragmatic trials. N Engl J Med. 2017;377:1391-8.

30. Ware $\mathrm{JH}$. Interpreting incomplete data in studies of diet and weight loss, N Engl J Med. 2003;348:2136-7.

31. Moher D, Hopewell S, Schulz KF, et al. CONSORT 2010 explanation and elaboration: updated guidelines for reporting parallel group randomised trials. Int J Surg. 2012;10:28-55.

32. Christensen R, Bliddal H, Henriksen M. Enhancing the reporting and transparency of rheumatology research: a guide to reporting guidelines. Arthritis Res Ther. 2013;15:109.

33. Vickers AJ, Altman DG. Analysing controlled trials with baseline and follow up measurements. BMJ. 2001;323:1123-4.

34. Karstoft $\mathrm{K}$, Winding $\mathrm{K}$, Knudsen SH, et al. The effects of free-living interval-walking training on glycemic control, body composition, and physical fitness in type 2 diabetic patients. Diabetes Care. 2013;36:228-36.

35. Lindegaard B, Hansen T, Hvid T, et al. The effect of strength and endurance training on insulin sensitivity and fat distribution in human immunodeficiency virus-infected patients with lipodystrophy. J Clin Endocrinol Metab. 2008;93:3860-9.
36. Helgerud J, Høydal K, Wang E, et al. Aerobic high-intensity intervals improve $\dot{V}_{2 \text { max }}$ more than moderate training. Med Sci Sports Exerc. 2007;39:665-71.

37. World Medical Association. World Medical Association Declaration of Helsinki Ethical Principles for Medical Research Involving Human Subjects. JAMA. 2013;310:2191-4.

38. Chan AW, Tetzlaff JM, Gøtzsche PC, et al. SPIRIT 2013 explanation and elaboration: guidance for protocols of clinical trials. BMJ. 2013;346:e7586.

\section{Ready to submit your research? Choose BMC and benefit from:}

- fast, convenient online submission

- thorough peer review by experienced researchers in your field

- rapid publication on acceptance

- support for research data, including large and complex data types

- gold Open Access which fosters wider collaboration and increased citations

- maximum visibility for your research: over $100 \mathrm{M}$ website views per year

At BMC, research is always in progress.

Learn more biomedcentral.com/submissions 